\title{
Deep generative models for fast shower simulation in ATLAS
}

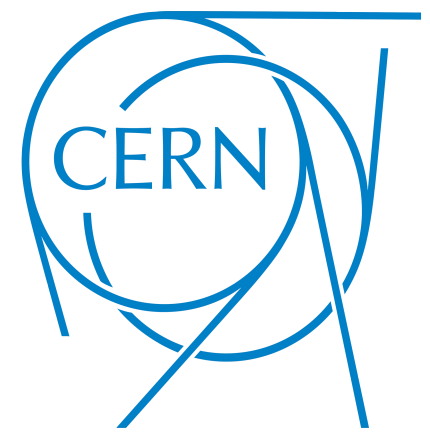

\section{Dalila Salamani}

University of Geneva

dalila.salamani@cern.ch

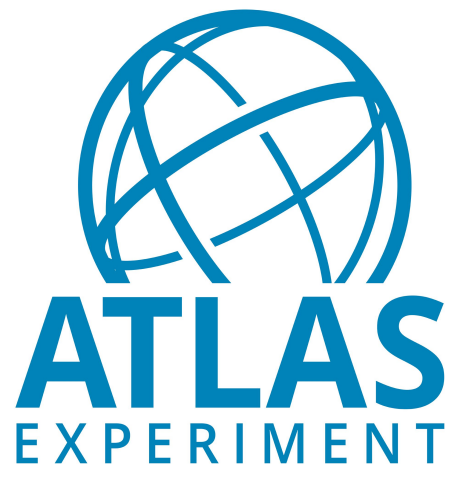

On behalf of the ATLAS collaboration

14th e-science IEEE International Conference 2018 


\section{Outline}

- Context \& Motivation

- Rise of generative models

- Generative models for HEP

- Model architectures : VAE \& GAN

- Validation of generation performance

- Conclusion 


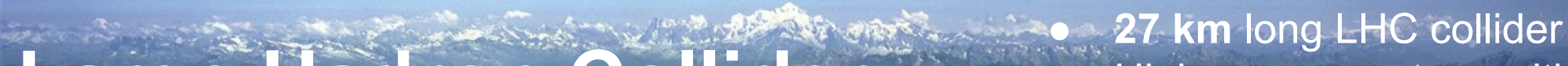

Large Hadron Collider

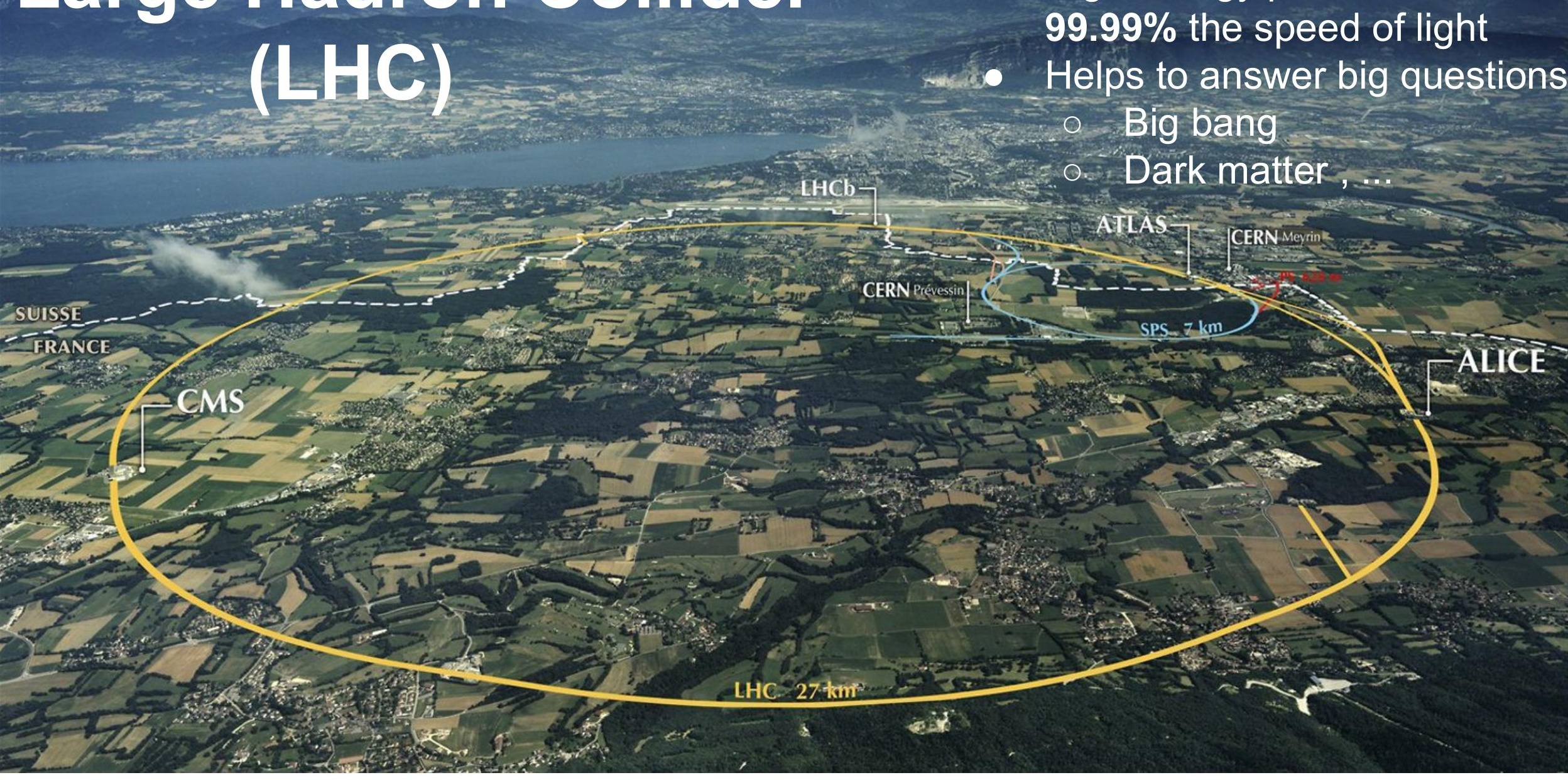

High energy protons with $99.99 \%$ the speed of light ips to answer big questions - Big bang

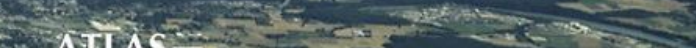




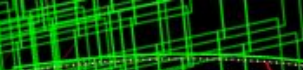 \\ Collision event}

7 i 1 and

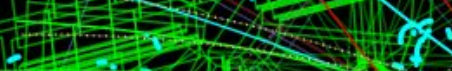

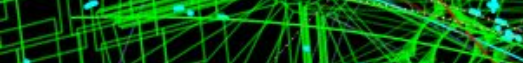

\section{,

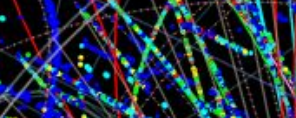

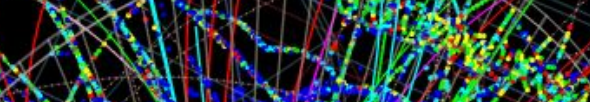

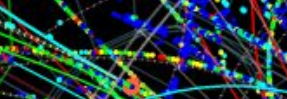

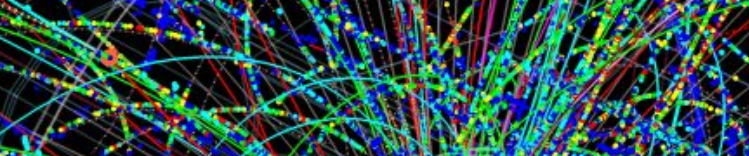

S.

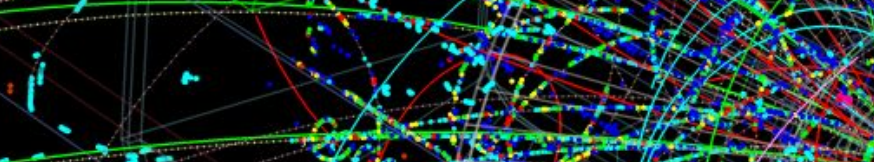

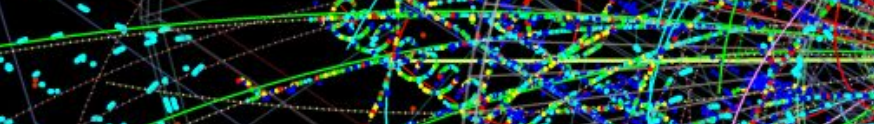

r...

$\therefore$ A
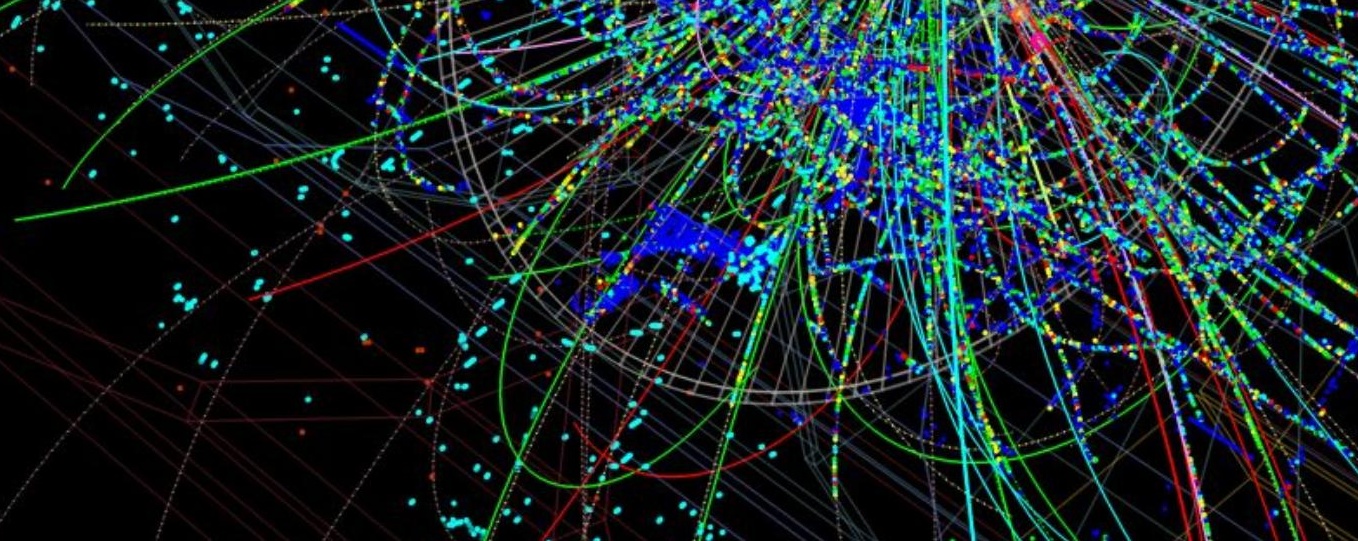


\section{ATLAS detector}

mion

spectrometer

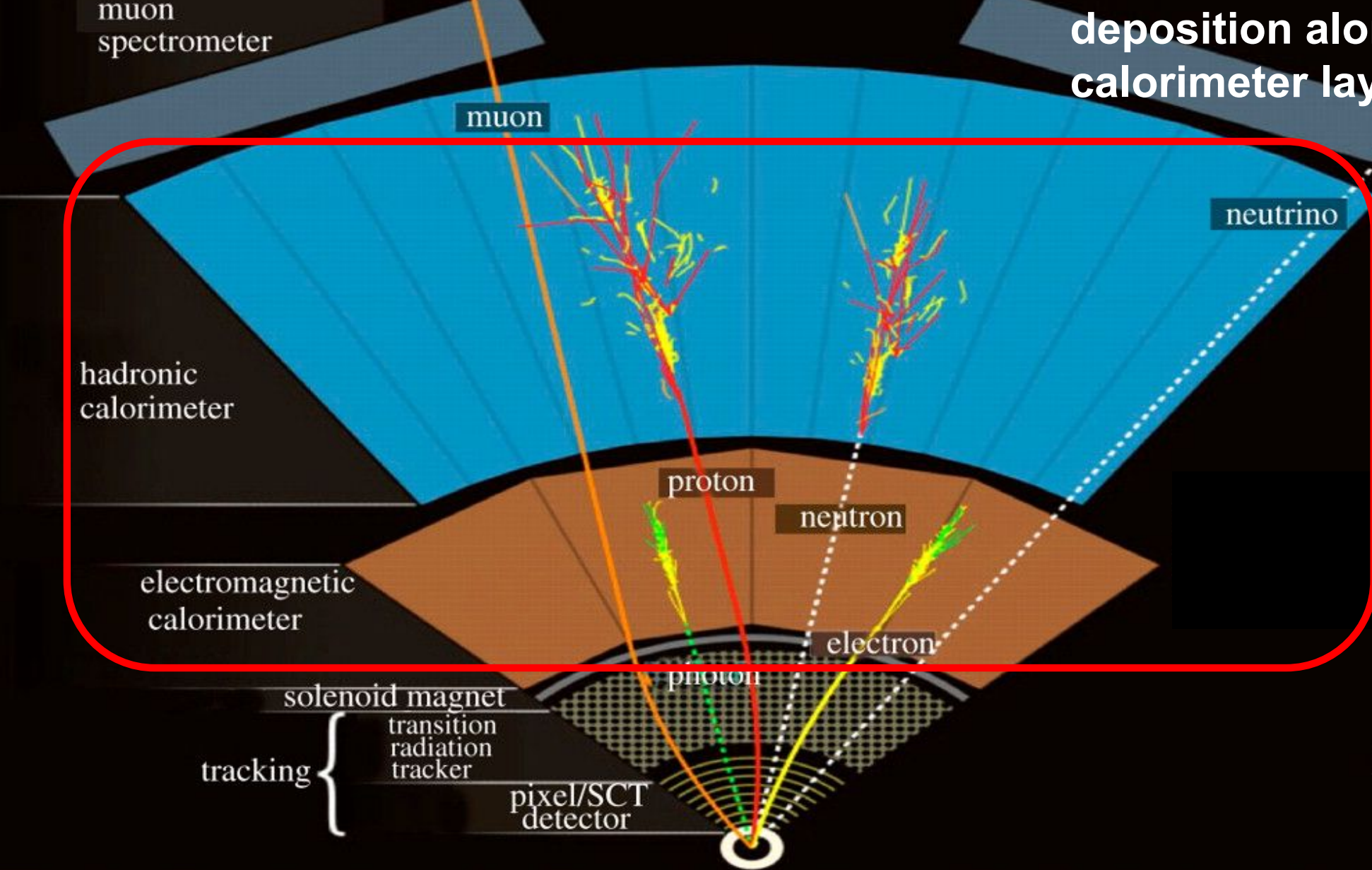

- Showering process Cascade of energy deposition along the yers: 


\section{ATLAS detector}

\section{thas d}

spectrometer

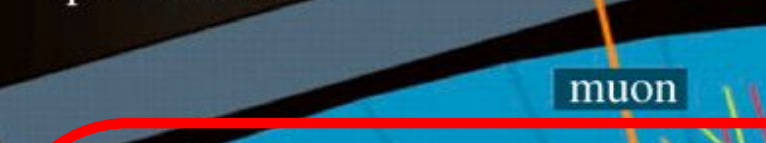

- Showering process

- Cascade of energy deposition along the calorimeter layers,
Detector simulation (MonteCarlo)

If'

hadronic

calorimeter

\section{FCC}

- Data analysis

electromagnetic

calorimeter

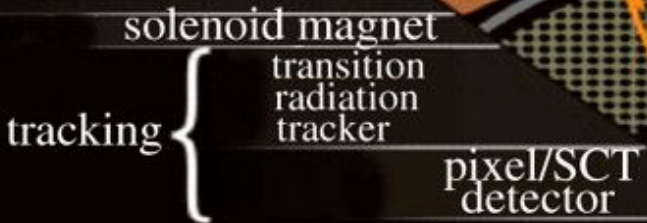

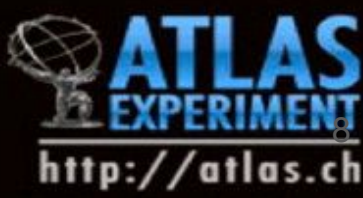




\section{Motivation}

- Successful physics program in ATLAS depends on the availability of high statistics Monte Carlo simulated events.

- Currently $>50 \%$ of ATLAS computing time is spent on shower simulation.

- $\mathrm{LHC}$ is collecting more and more events (High Luminosity Upgrade) $\rightarrow$ more CPU consumption.

CPU consumption All Jobs in seconds (Sum: 7,263,679,689,134) MC Simulation - $64.89 \%$

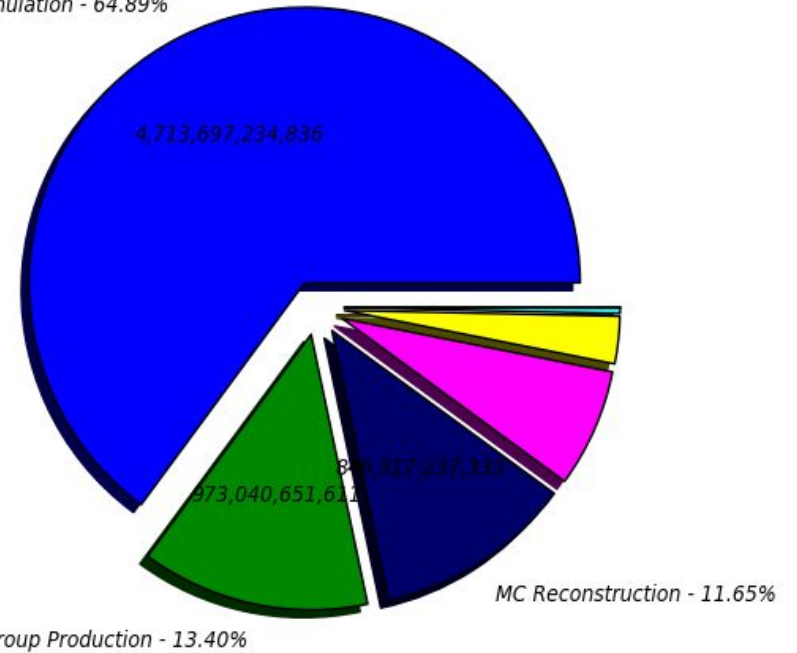

Group Production - $13.40 \%$
MC Simulation - $64.89 \%(4,713,697,234,836)$
- MC Reconstruction - $11.65 \%(846,317,237,333)$ MC Reconstruction - $11.65 \%(846,317,237,33$
Data Processing - $2.77 \%(201,387,396,932)$
unknowin $-0.00 \%(0.00)$
- Group Production - $13.40 \%(973,040,651,611)$ Analysis - $6.95 \%$ (504, $771,330,24)$ Others $-0.34 \%(24,465,838,17$
To Processing $-0.00 \%(0.00)$

Reference 


\section{Motivation}

- Successful physics program in ATLAS depends on the availability of high statistics Monte Carlo simulated events.

- Currently $>50 \%$ of ATLAS computing time is spent on shower simulation.

- $\mathrm{LHC}$ is collecting more and more events (High Luminosity Upgrade) $\rightarrow$ more CPU consumption.

- MC Simulation - $64.89 \%(4,713,697,234,836)$ $\square$ Data Processing - $2.77 \%(201,387,396,932)$ unknown - $0.00 \%(0.00)$
CPU consumption All Jobs in seconds (Sum: 7,263,679,689,134) MC Simulation - $64.89 \%$

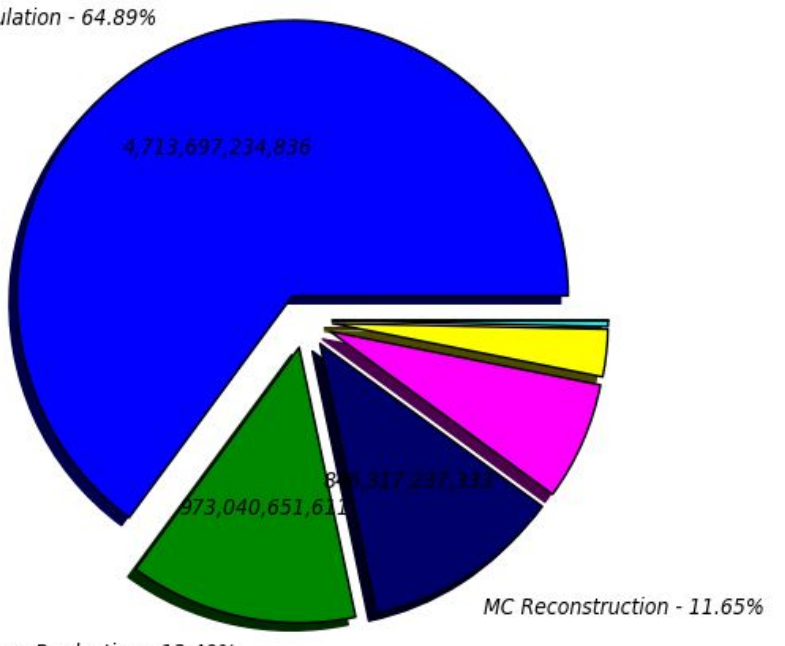
Group Production - $13.40 \%$

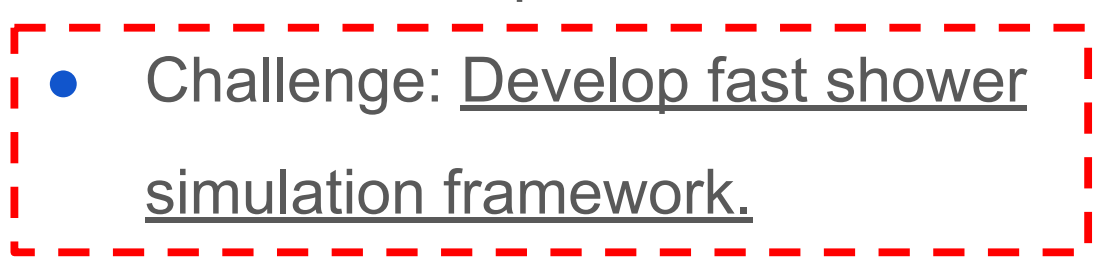

Group Production - $13.40 \%(973,040,651,611$ Analysis - $6.95 \%(504,771,330,247)$ - Others - $0.34 \%(24,465,838,17)$ 


\section{Machine learning}

- Learning process

- Learn to improve performance by experience *

- Automatic \& Adapted model to domain application

- Discover knowledge from dataset (engineering bottleneck )

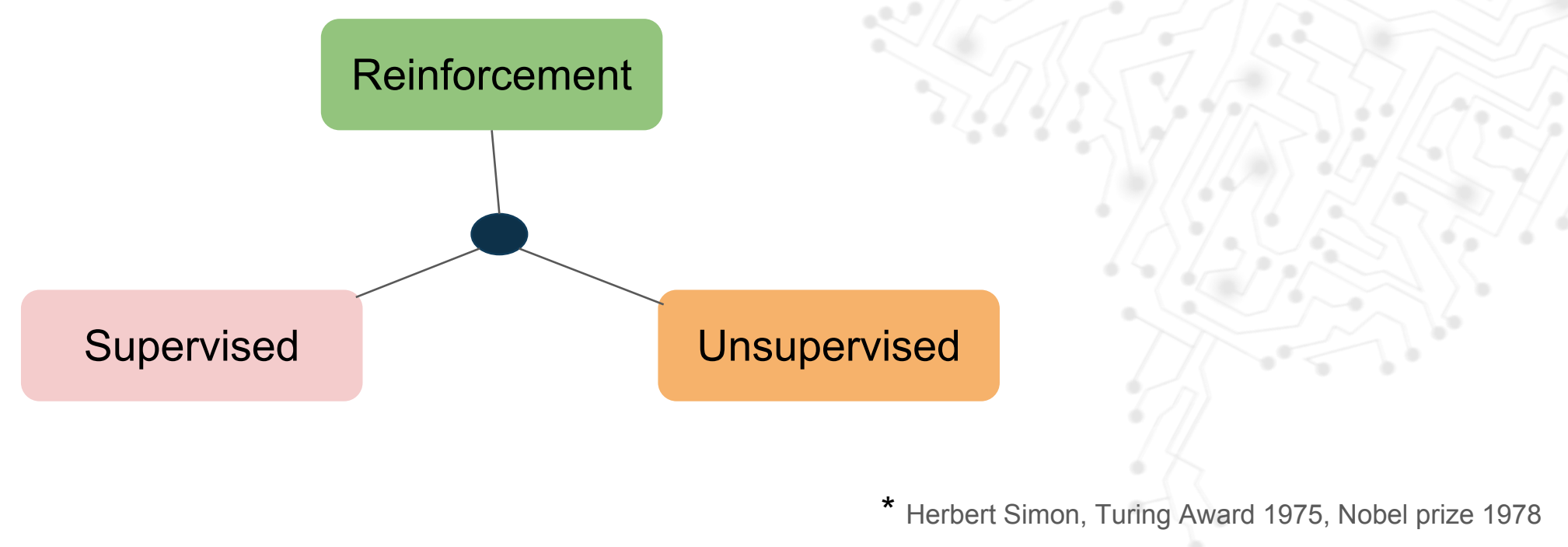




\section{Machine learning}

- Learning process

- Learn to improve performance by experience *

- Automatic \& Adapted model to domain application

$\circ \quad$ Discover knowledge from dataset (engineering bottleneck )

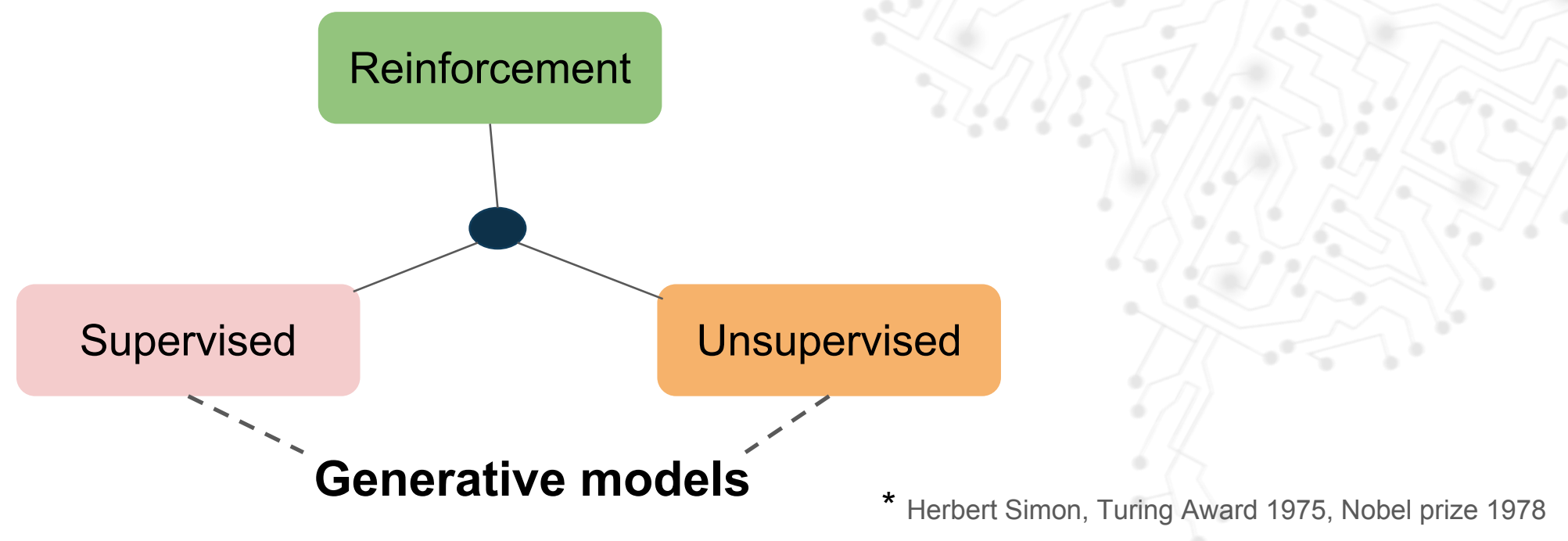




\section{Generative models}

- Learn the true data distribution of the training set to reproduce it.

- Adopted approaches: Use of deep neural networks to learn the approximation function of the true (\& sparse) distribution, Variational Autoencoders (VAEs) \& Generative Adversarial Networks(GANs)

Noise $\sim \mathrm{N}(0,1)$

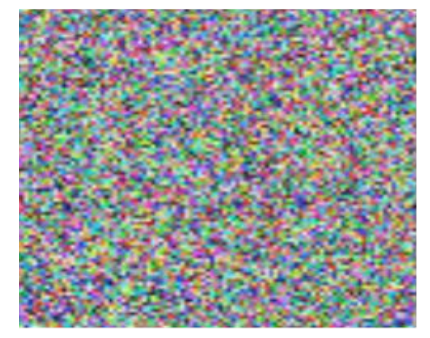

\section{Generate}

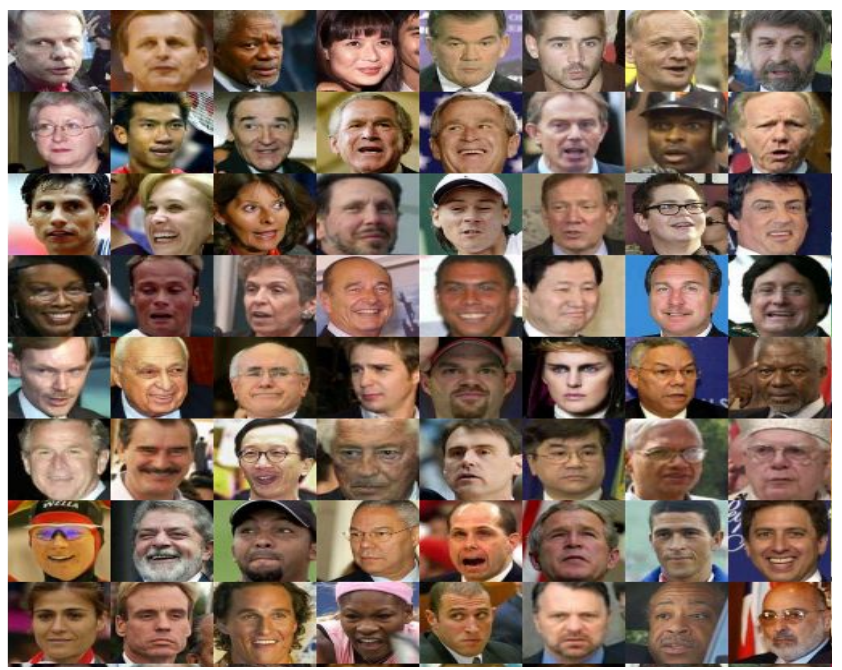




\section{Generative models : domain application}

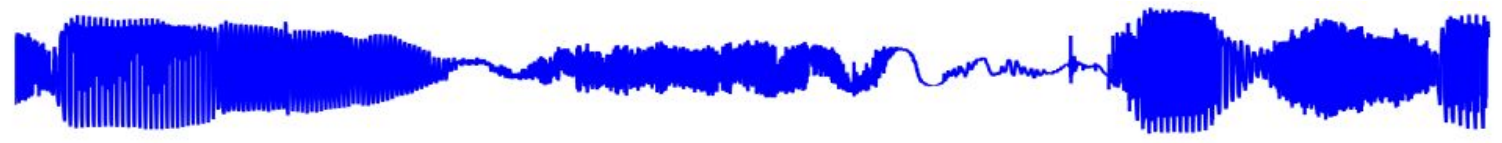

Learning to generate speech : Den Oord et al, 2016

Drug Discovery : $\underline{\text { Chen et al, } 2018}$
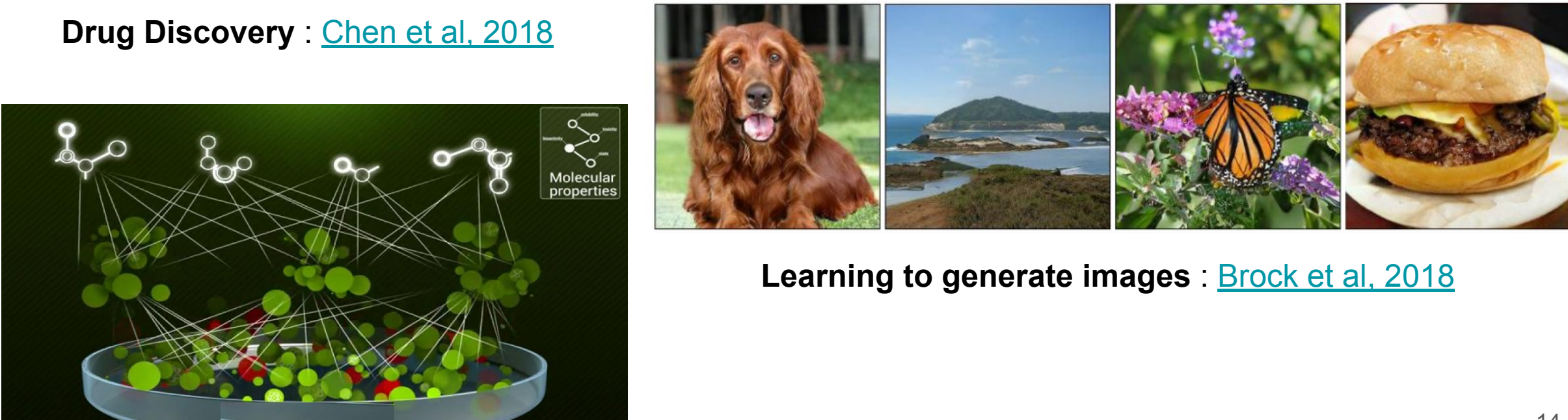

Learning to generate images : Brock et al, 2018 


\section{Generative models for HEP (Showering)}

- Model the shower process.

- Take into account the ATLAS calorimeter geometry.

- Validation : shower shape variables distribution comparison.

- Fast \& accurate modeling.

- First application of deep generative models for fast shower simulation in ATLAS: Public Note ATL-SOFT-PUB-2018-001. 


\section{Dataset \& preprocessing}

- Single photon samples in the electromagnetic.

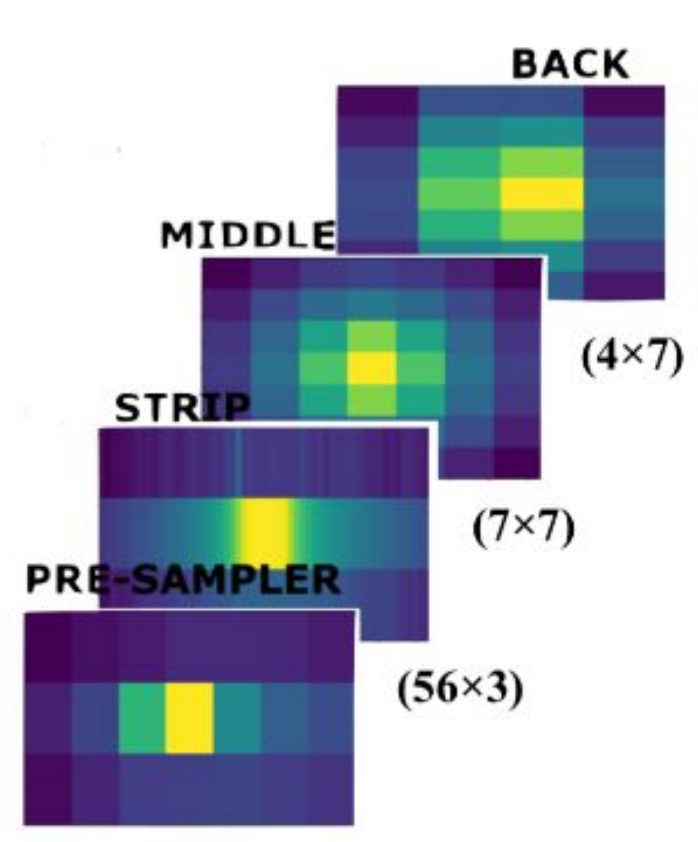

$(7 \times 3)$

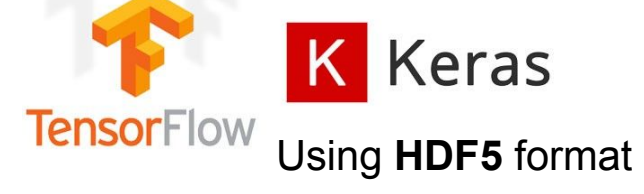




\section{VAE model architecture}

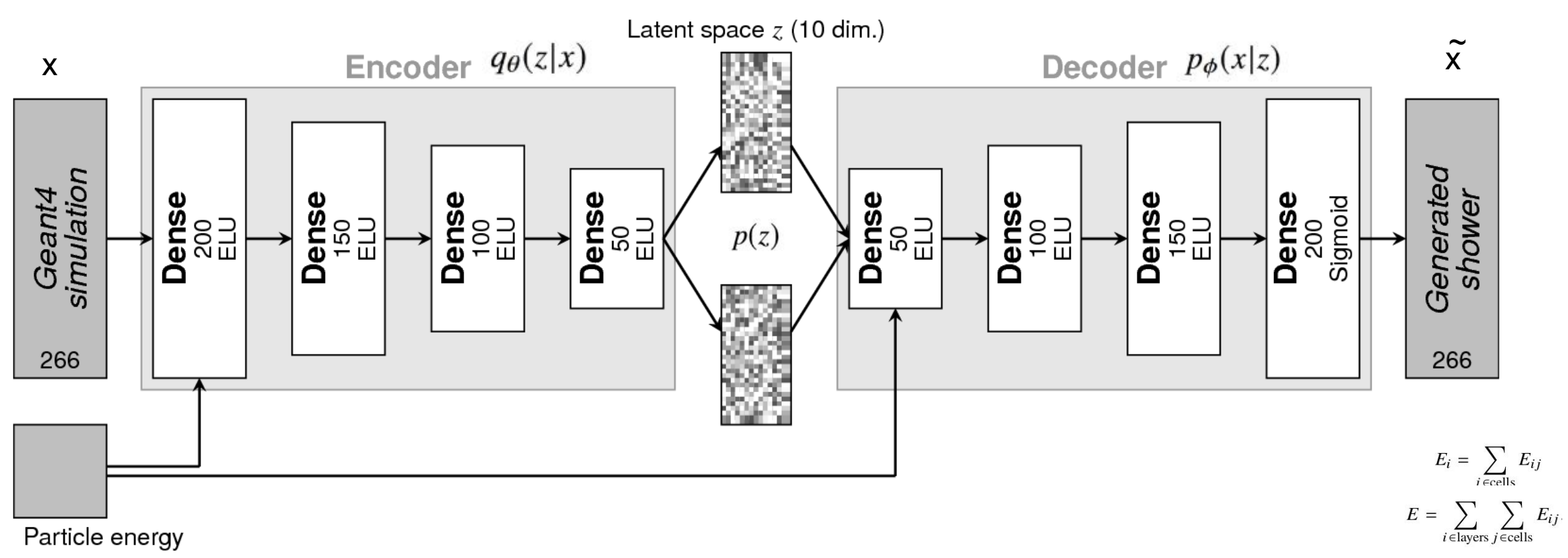

$$
L_{\mathrm{VAE}}(x, \tilde{x})=w_{\mathrm{reco}} E_{z \sim q_{\theta}(z \mid x)}\left[\log p_{\phi}(x \mid z)\right]-w_{\mathrm{KL}} \mathrm{KL}\left(q_{\theta}(z \mid x) \| p(z)\right)+w_{E_{\mathrm{tot}}} L_{E_{\mathrm{tot}}}(x, \tilde{x})+\sum_{\substack{i \\ \text { Total Energy fraction per layer } \\ \text { Loss }}}^{M} w_{i} L_{E_{i}}(x, \tilde{x})
$$




\section{GAN model architecture}

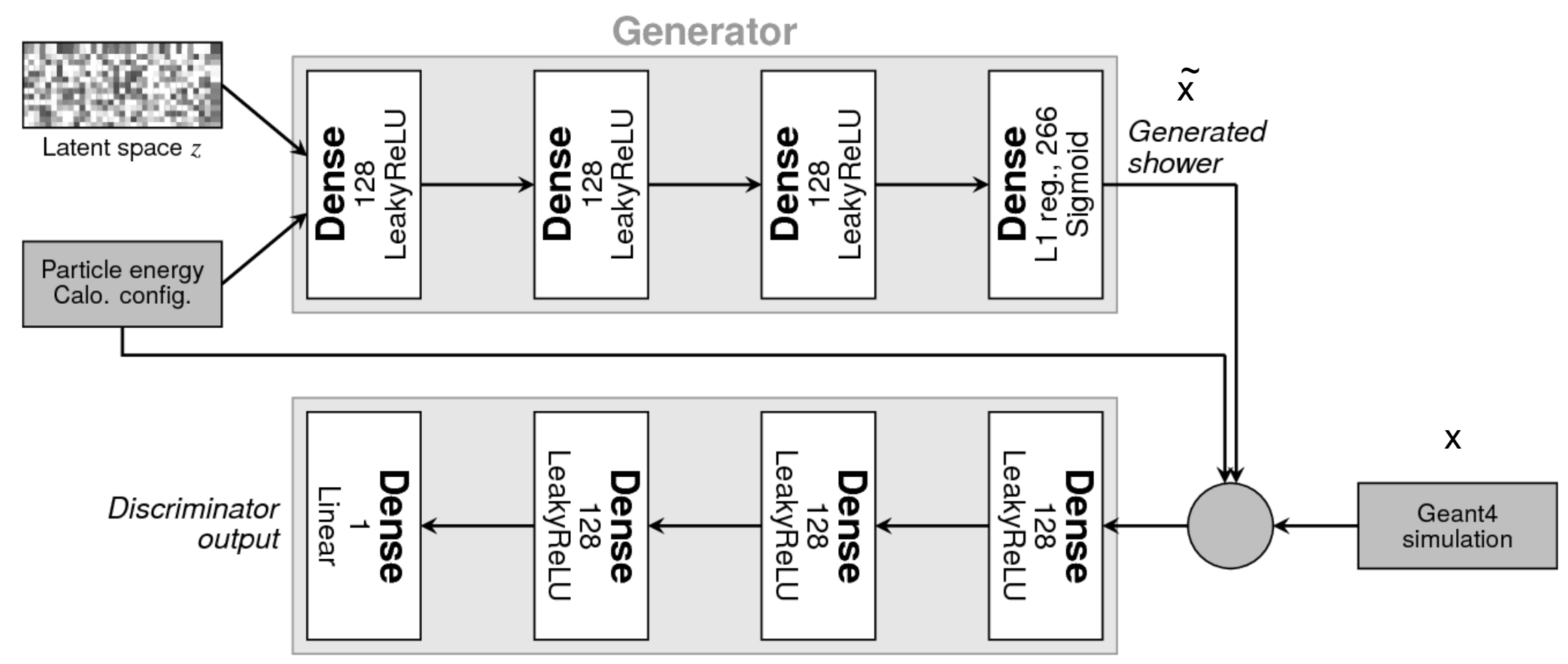

\section{Discriminator}

$$
L_{\mathrm{GAN}}=\underset{\tilde{x} \sim p_{\text {gen }}}{E}[D(\tilde{x})]-\underset{x \sim p_{\text {Geant } 4}}{E}[D(x)]+\underset{\hat{x} \sim p_{\hat{x}}}{E}\left[\left(\left\|\Delta_{\hat{x}} D(\hat{x})\right\|_{2}-1\right)^{2}\right]
$$




\section{Generation results: energy per layer}

- Energy deposited in the individual electromagnetic calorimeter layers for photons $65 \mathrm{GeV}$.

- Challenges posed by layers with low (and sparse) energy deposits, i.e. late showers.
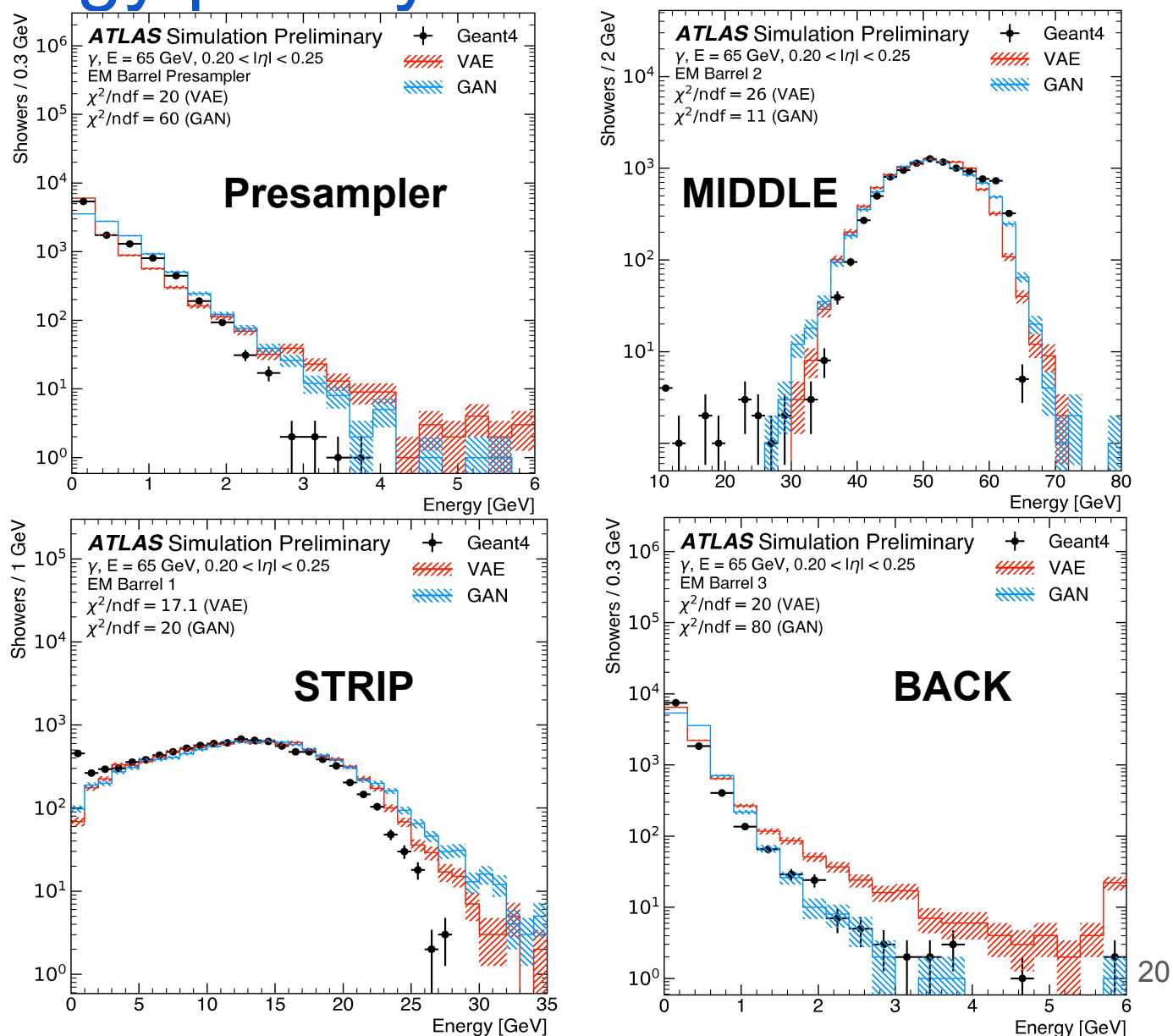


\section{Generation results: Average energy vs $\Delta \eta, \Delta \phi$}

\section{Average energy vs $\Delta \eta$ Layer : STRIP}

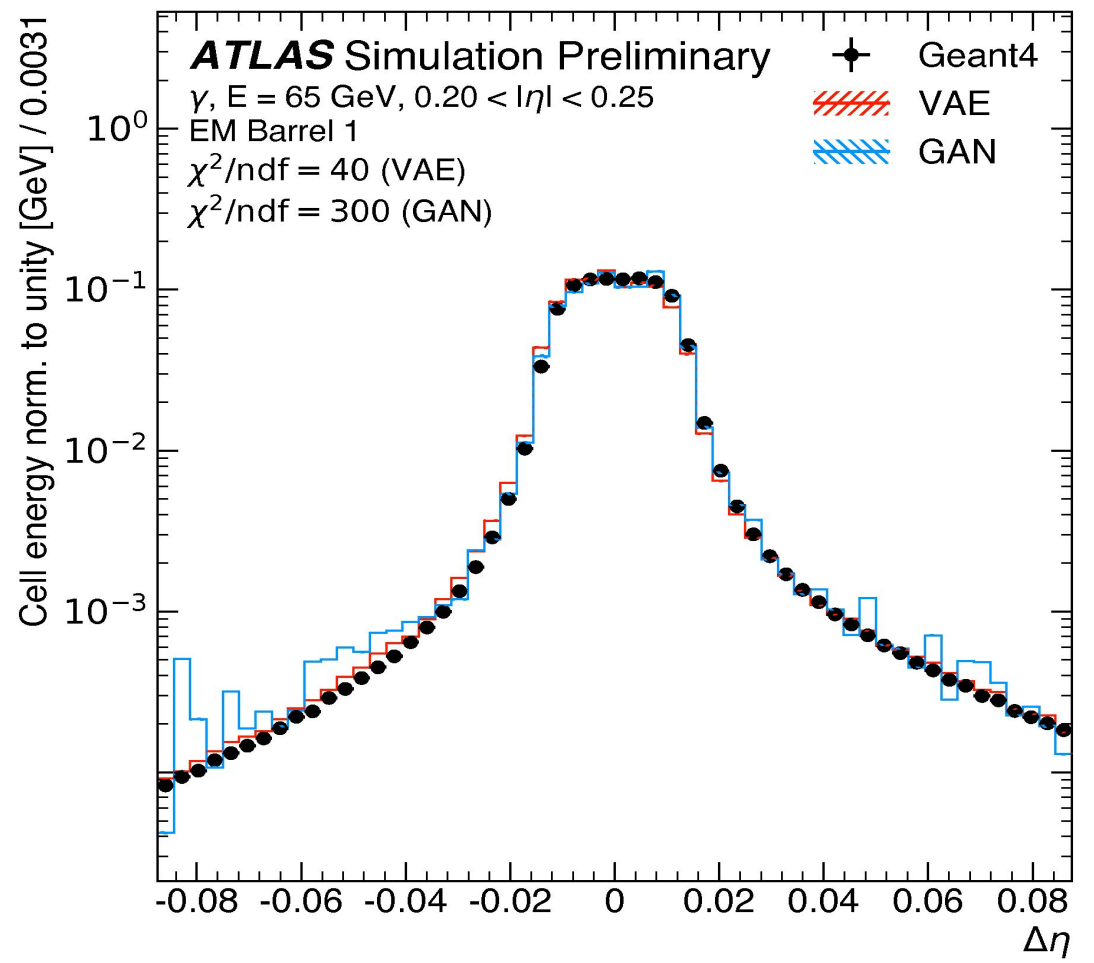

Average energy vs $\Delta \phi$ Layer : MIDDLE

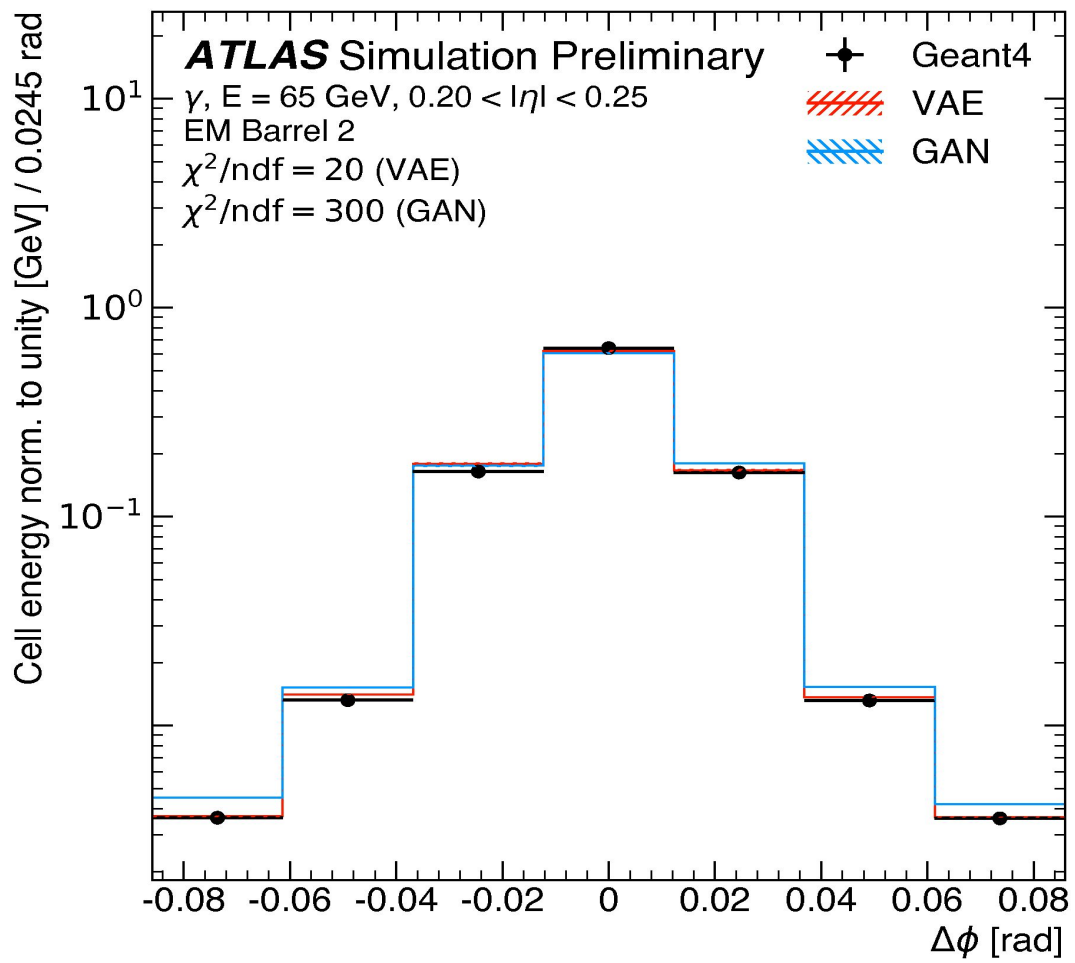




\section{Conclusion \& Outlook}

- Fast shower simulation is essential for LHC experiments physics program.

- Proof of concept for generative Deep Learning models for simulating particle showers.

- Promising results and active developement towards achieving required accuracy.

- Outlook: improve the model to fit a larger class of particle types \& pseudorapidity regions. 


\section{Backup slides}




\section{Hyperparameters optimization for VAE}

\begin{tabular}{ll}
\hline \hline Hyperparameter & Values \\
\hline Latent space dim. & {$[1, \ldots, \mathbf{1 0}, \ldots, 100]$} \\
Reco. weight & $(0, \ldots, \mathbf{1}, \ldots, 3]$ \\
KL weight & $(0, \ldots, \mathbf{1 0}-\mathbf{- 4}, \ldots, 1]$ \\
$E_{\text {tot weight }}$ & {$\left[0, \ldots, \mathbf{1 0} \mathbf{-}^{-\mathbf{2}}, \ldots, 1\right]$} \\
& {$\left[0, \ldots, \mathbf{8} \times \mathbf{1 0}^{-\mathbf{2}}, \ldots, 1\right]$} \\
$E_{i}$ weights & {$\left[0, \ldots, \mathbf{6} \times \mathbf{1 0}^{-\mathbf{1}}, \ldots, 1\right]$} \\
& {$\left[0, \ldots, \mathbf{2} \times \mathbf{1 0}^{-\mathbf{1}}, \ldots, 1\right]$} \\
& {$[0, \ldots, \mathbf{1 0}, \ldots, \ldots, 1]$} \\
Hidden layers (encoder) & $1,2,3, \mathbf{4}, 5$ \\
Hidden layers (decoder) & $1,2,3, \mathbf{4}, 5$ \\
& {$[180, \ldots, \mathbf{2 0 0}, \ldots, 266]$} \\
Units per layer & {$[120, \ldots, \mathbf{1 5 0}, \ldots, 180]$} \\
& {$[80, \ldots, \mathbf{1 0 0}, \ldots, 120]$} \\
Activation func. & {$[10, \ldots, \mathbf{5 0}, \ldots, 80]$} \\
Kernel init. & ELU, ReLU, SELU, LeakyReLU, PReLU \\
& zeros, ones, random normal, random uniform, truncated normal, \\
Bias init. & variance scaling, glorot_normal \\
Optimizer & zeros, ones, random normal, random uniform, truncated normal, \\
Learning rate & variance scaling, glorot_normal \\
Mini-batch size & RMSprop, Adam, Adagrad, Adadelta, Nadam \\
\hline \hline
\end{tabular}




\section{Hyperparameters optimization for GAN}

\begin{tabular}{|c|c|}
\hline Hyperparameter & Values \\
\hline Hidden layers & $1, \mathbf{3}, 5,10$ \\
\hline Units per layer & $64, \mathbf{1 2 8}, 512,1024$ \\
\hline Activation func. & $\begin{array}{l}\text { SELU + Sigmoid, LeakyReLU }+\{\text { Sigmoid, ReLU, } \\
\text { Gauss, Sigmoid + ReLU, clipped ReLU, softmax, } \\
\text { softmax + ReLU }\}\end{array}$ \\
\hline Activity L1_REG_WEIGHT (Gen.) & $0,10^{-5}, 10^{-2}$ \\
\hline Kernel init. & glorot_uniform, lecun_normal \\
\hline Gradient penalty & one-sided, two-sided \\
\hline Gradient penalty weight & $0, \mathbf{1 0}, 20$ \\
\hline Training ratio & $\begin{array}{l}20,10, \mathbf{5}, 3,1 \\
\mathbf{5} \times \mathbf{1 0}^{-\mathbf{5}}, 5 \times 10^{-6} 1 \times 10^{-6}(\text { trainino ratio } 5)\end{array}$ \\
\hline Learning rate & $\begin{array}{l}\left.5 \times 10^{-5}, 5 \times 10^{-6}, 1 \times 10^{-5}, 1 \times 10^{-7} \text { (training ratio } 3\right) \\
1 \times 10^{-6}(\text { training ratio } 1)\end{array}$ \\
\hline Mini-batch size & 64, 1024 \\
\hline $\begin{array}{l}\text { Preprocessing (all norm. to } E_{\gamma} \text { ) } \\
\text { Conditioning }\end{array}$ & $\begin{array}{l}\log _{10} E_{\text {cell }}, \log _{10}\left(E_{\text {cell }} \times 10^{10}\right), E_{\text {cell }} \\
\left\{E_{\gamma}, \log _{10} E_{\gamma}\right\}+\text { multi-hot encoding of cell alignments }\end{array}$ \\
\hline
\end{tabular}

